\title{
La cultura corporativa: una revisión crítica desde la Sociología del trabajo
}

\author{
Szlechter, Diego*
}

\section{Resumen}

El debate acerca de los aspectos simbólicos del trabajo surge a mediados de la década del 70 , como respuesta al paradigma teórico estructuralista imperante desde la 2da. Guerra Mundial. Al mismo tiempo, comienzan a aflorar perspectivas manageriales preocupadas en la consecución de una cultura organizacional homogénea, generalmente consustanciadas con las altas direcciones de las grandes firmas. En este artículo, se abordará de manera crítica el surgimiento de la preocupación por la cultura de empresa en el debate académico a través de un relevamiento de la literatura proveniente de la Sociología del trabajo contrastada con fuentes bibliográficas provenientes del ámbito del Management y de la Sociología de las organizaciones. De esta manera, se logra develar el carácter eminentemente ideológico de los enfoques managerialistas de la cultura, inspirados esencialmente de la llamada "doctrina de la excelencia", revelando las contradicciones en las que incurre la retórica empresarial al abordar la cuestión de la cultura, apelando a la búsqueda de "significados compartidos" al tiempo que intenta concitar el compromiso, la movilización y la lealtad de sus empleados con la cultura de la firma. Como conclusión se observan las consecuencias directas de las políticas de recursos humanos tendientes a encuadrar a los empleados con la cultura organizacional, llevando a una gestión híbrida y dual de la fuerza de trabajo, jerarquizando a la misma y en la cual habrá ganadores y perdedores.

Palabras clave: Cultura corporativa, management, sociología del trabajo.

Recibido: 30-06-12. Aceptado: 07-11-13

* Investigador-docente, Instituto de Industria, Universidad Nacional de General Sarmiento y CONICET. Buenos Aires, Argentina, e-mail: diego_szlechter@yahoo.com.ar 


\title{
Corporate Culture: A Critical Review Seen from the Sociology of Work
}

\begin{abstract}
The debate about the symbolic aspects of work arose in the mid 1970's, in response to the structuralist theoretical paradigm prevailing since World War II. At the same time, managerial perspectives began to emerge, concerned with achieving a homogeneous organizational culture, generally consubstantial with the top management of large companies. This article takes a critical approach to the emergence of concern for the culture of enterprise in academic debate by revealing literature from the sociology of work contrasted with bibliographic sources from the managerial realm and the sociology of organizations. In this way, the eminently ideological character of the managerial approaches to culture, inspired essentially by the so-called "doctrine of excellence," is unveiled, revealing contradictions in what managerial rhetoric incurs when addressing the issue of culture, invoking the search for "shared meanings" while it attempts to incite compromise, mobilization and loyalty in its employees to the firm's culture. The conclusion observes the direct consequences of human resource policies that tend to frame employees within the organizational culture, leading to hybrid and dual management of the work force, hierarchizing it and creating a situation in which there will be winners and losers.
\end{abstract}

Keywords: Corporate culture, management, sociology of work.

\section{Introducción}

En este trabajo se abordará la emergencia de la preocupación por la llamada cultura organizacional en la literatura cercana a concepciones manageriales, como ser el campo de la Administración (en sus variantes de las Ciencias de la gestión y del Management) y la Sociología de las organizaciones. El objetivo del análisis del surgimiento de este fenómeno es para contrastarlo con las vertientes que manan de la Sociología del trabajo, preocupadas por los aspectos simbólicos del proceso de trabajo y con posiciones críticas de las corrientes que parten de la posibilidad de una convivencia armónica entre el capital y el trabajo, tal como lo hace el managerialismo (Deetz, 1992).

En este sentido, se intenta territorializar el debate al analizar la incorporación de los análisis culturalistas en nuestra región, especialmente en la Argentina. Si bien este trabajo no deja de ser una revisión crítica de la literatura, se apoya en fuentes secundarias de una de las consultoras más importantes del mundo: Great place to work ${ }^{1}$, dedicada a verificar el grado de alineamiento de los emplea-

1 Actualmente está presente en 40 países. Se proponen como misión la de "construir una sociedad mejor ayudando a las empresas a transformar sus ambientes de trabajo". Una de sus mayores fuentes de ingreso lo constituye la publicación de un ranking de los 100 mejores lugares para trabajar, en el cual figuran las empresas más grandes del país, la mayoría de ella grupos transnacionales (Great Place to work, 2011). 
dos con los objetivos empresariales de las firmas más grandes del mundo. Esta consultora tiene su sede en la Argentina y realiza una encuesta anual sobre "clima organizacional" que releva datos de las 100 compañías de mayor facturación en el país. Esto ayudará a brindar un clivaje territorial al relevamiento de la literatura managerial dado su sesgo etnocentrista, que no hace más que reproducir las modas que campean en los centros del poder económico mundial. La contrastación de las reflexiones de los campos mencionados, nos ayudará a dilucidar las realidades que esconde una bibliografía que se desentiende del conflicto siempre latente al interior de las relaciones de poder de las organizaciones.

\section{Emergencia de la cuestión cultural en los estudios del trabajo: breve derrotero histórico}

Dentro de la Sociología del Trabajo, el debate acerca de los aspectos simbólicos y significativos del trabajo surge a mediados de la década del 70 del Siglo pasado, como respuesta al paradigma teórico estructuralista imperante desde la Segunda Guerra Mundial. En este período, la Sociología en general y la Sociología del trabajo en particular (y en sus variantes de los "Labour studies" en Gran Bretaña y las "Industrial relations" en los EEUU) comienza a indagarse acerca del contenido simbólico del proceso de trabajo, la construcción de las identidades laborales y la ideología de la empresa. El proceso de modernización industrial y de urbanización de la Posguerra hizo surgir la preocupación en torno al destino y la adaptación del hombre frente al vertiginoso cambio industrial y tecnológico (López, 1986; Brown, 1982). Con enfoques teóricos prestados desde diversas disciplinas como la Antropología y la Psicología, se comienza a indagar acerca de temas tales como el contenido simbólico del proceso de trabajo, la orientación de los individuos hacia el trabajo, la construcción de las identidades laborales y la ideología de la empresa.

Uno de los países precursores en abordar esta problemática fue Francia, cuyos debates dentro del campo de la Sociología del trabajo y de las calificaciones han sido fructíferos al encarar estudios sobre los efectos de la denominada modernización empresaria en las nuevas demandas de calificaciones y competencias, en los sistemas de calificación profesional y en los modelos de profesionalización. Sin embargo, resulta menos nítida la preocupación por la reconfiguración del espacio cultural/político, como lugar sustancial de recomposición del orden hegemónico empresario (Fígari, 2007).

Junto con la preocupación por las relaciones simbólicas que subyacen a las relaciones de producción desde la Sociología del trabajo, surgen corrientes de pensamiento con una fuerte impronta apologética de la llamada cultura corporativa (Deal y Kennedy, 1982; Peters y Waterman, 1986; Calas y Smircich, 1992; Schein, 1995). Estos postulados desconocían el carácter político -conflictivo- de la cultura, centrándose exclusivamente en los aspectos que concitan adhesión de los empleados con la cultura de las compañías. Partiendo de postulados managerialistas, el debate en torno a la cultura corporativa u organizacional encuentra 
su fuente de inspiración en la teoría funcionalista (De Gaulejac, 2005) con su visión comportamentalista del individuo y su consideración reificada de la organización, tomándola como un sistema, una "caja negra", una entidad que ha de tener un funcionamiento "normal" y cuya finalidad exclusiva es la de asegurar su reproducción. Esta perspectiva, más normativa que explicativa, más adaptativa que comprehensiva, se apoya sobre los modelos de la física, la cibernética y la biología, buscando medir los desvíos respecto de la norma, calcular los niveles de desarrollo y de crecimiento que se deben conseguir en función de ciclos y de parámetros preconstruidos con el fin de indicar los niveles óptimos.

Dentro de una postura más matizada que se nutre de elementos tanto de la Sociología crítica del trabajo -especialmente francesa- como de corrientes managerialistas, en los Estados Unidos, el enfoque cultural de la Sociología industrial (llamada comúnmente Industrial relations) y del trabajo se nutrió del interaccionismo ${ }^{2}$ simbólico de la Escuela de Chicago de los años 20 y 30 del siglo pasado, aunque también recogió elementos importantes de la Teoría weberiana de la acción social (bajo el prisma parsoniano) y del análisis estructuralista sobre las ocupaciones y las profesiones (Watson, 1987: 28-59). Trabajos como el de Hughes (1958) iban por ese camino, aunque su interés como el de sus colegas de Chicago, estaba centrado en situaciones de trabajo de los "inadaptados" al sistema.

Varios años después, la Sociología de las organizaciones comenzará a inspirarse en estas ideas para estudiar las interacciones y adaptaciones entre las distintas "subculturas" que se mueven dentro de las organizaciones, ya sea de grupos ocupacionales o la misma cultura gerencial. El enfoque elegido para dichas investigaciones será el estructural - funcionalista parsoniano (Ibarra, 2000: 255), por lo que el "contexto" (macrosocial y no el organizacional) pasa a ser dejado de lado. Desde una perspectiva parsoniana, la cultura organizacional es mirada como un objeto en sí mismo con sus particularidades específicas 0 , en otras palabras, sistemas institucionalizados de valores que orientan la acción. Se hace hincapié en la consistencia, la armonía, el orden y el consenso, negando el conflicto de intereses o la presencia de otras culturas. Sólo así se puede comprender la aceptación que tuvo la Escuela de las relaciones humanas de EEUU a partir de la década del 30 , la cual sostenía que la productividad de la organización era consecuencia de la cohesión social dentro de la misma.

Durante los últimos años, la Sociología de las organizaciones ha seguido abordando de manera profusa la cuestión de la cultura organizacional. De acuerdo a Braga (1996), en contraste con las décadas del 60 y 70 , donde la atención estaba puesta en el comportamiento utilitarista racional de los

2 El interaccionismo pone su interés en procesos de interacción de los individuos, dejando en un segundo plano aspectos externos, estructurales o de condiciones materiales de posibilidad que determinan las conductas de los mismos. 
actores al interior de las organizaciones, recientes estudios se han enfocado en la subjetividad y las emociones puestas en juego en la vida organizacional. Esto puede deberse al fracaso de las empresas occidentales en reproducir los niveles de productividad japoneses al trasplantar sus técnicas de management, lo cual revelaba que en realidad dichas técnicas se encuentran encastradas en la cultura que les da origen.

Sin apartarse de las perspectivas que buscan encontrar la verdadera cultura de una empresa, Deal y Kennedy (1982) ubican al surgimiento de la preocupación por la vinculación entre el comportamiento del individuo y las estructuras de la organización como fundamento del debate sobre cultura organizacional. De acuerdo a estos autores, el comportamiento será abordado desde la perspectiva de los valores y las creencias que propician la unidad y la integración en torno a la organización.

De todas maneras, los trabajos al interior de la Sociología de las organizaciones y de las perspectivas abiertamente managerialistas -y etnocéntricas- parten de supuestos teóricos cuya raíz se funda en la convivencia armónica entre capital y trabajo, enfatizando el carácter homogéneo de la cultura de empresa. En efecto, ésta siempre es mostrada desde su capacidad de integración y su poder para crear significados compartidos más que de generar resistencia entre los miembros que la componen. El director de la consultora Great Place to Work (GPTW) -con sede en EEUU y filiales en todo el mundo, incluyendo a la Argentinacomprende muy bien la necesidad de una convivencia armónica entre el asalariado y los altos directivos. Así lo señala en la página web institucional de la organización:

... porque cuando uno habla de clima organizacional está hablando básicamente de liderazgo, de trabajo en equipo, de confianza, de un montón de elementos que aglutinan y que hacen a un todo, una organización (Great place to work, 2011).

Dentro del debate latinoamericano, los estudios culturales sobre el trabajo tuvieron su punto de inflexión a mediados de los 70, al derrumbarse las teorías estructuralistas sobre el desarrollo latinoamericano (Sztulwark, 2002) y de las concepciones evolucionistas sobre la clase obrera. Surgen así dos enfoques que pondrán énfasis en el mismo sujeto colectivo: la clase obrera.

- Uno de estos campos de análisis será conocido como cultura obrera, cultura del trabajo o cultura laboral. Sin embargo, el foco sigue estando en la clase obrera, ya sea para analizar las condiciones socioproductivas de los procesos de trabajo las cuales ayudan a explicar las condiciones de existencia de los mismos dentro y fuera del trabajo o para indagar acerca de la acción obrera.

- Otro de los enfoques es el que se centra en la reproducción social de los individuos, circunscribiendo la relación entre factores estructurales y subjetivos que orientan a los individuos dentro y fuera del trabajo. Así, se analizan trayectorias de trabajo, identidades profesionales, culturas y subculturas ocupacionales, procesos de cualifica- 
ción y las filosofías gerenciales (Guadarrama, 2000: 224). Si bien este último enfoque podría haber incluido como objeto de estudio a los asalariados más calificados, en general las investigaciones también tomaron como punto de referencia sólo a la clase obrera.

La emergencia del debate en torno a la cultura corporativa en nuestro continente se nutre del segundo enfoque, encontrando sustento en el análisis que Abramo y Montero (2000) emprenden acerca de la forma que adopta la emergencia del actor empresario en Latinoamérica. A partir de la década del 80 , con la crisis de la deuda externa, la globalización de la economía y los cambios en los patrones internacionales de competitividad, se transforma radicalmente el contexto de la disciplina. Así, la economía en su versión tecnocrática le gana espacio a la política, por lo que el tema pasa a ser la medida del ajuste a los nuevos patrones hegemónicos a nivel internacional, para poder recuperar capacidad competitiva e insertarse nuevamente en "el mundo" y así volver a transitar la senda del crecimiento.

Este proceso posee una marcada influencia en la Sociología del trabajo de la región, la cual vuelve a poner el tema de la modernización en el centro de las discusiones, pero ya no identificada con la idea de cambio social sino con la adecuación necesaria a un nuevo paradigma, un best way "posfordista", "toyotista", ligado al lean production, a la empresa esbelta, desjerarquizada. Es de esta forma que comienzan a desaparecer los sujetos, especialmente la clase trabajadora, emergiendo el empresario como centro de la atención y de las investigaciones. Este empre- sario de la gran corporación globalizada, modernizada, inserta en la economía internacional, sale victorioso frente al Estado "elefantiásico" y la gran "burocracia sindical”. ¿Cuál sería la medida del éxito de este actor? La "imperiosa" adecuación a los nuevos patrones de competitividad dominantes a escala global.

Para que todo esto tenga sentido al interior de las empresas, será necesario hacer hincapié en la identificación de los asalariados con el nuevo patrón de competitividad global. El manager pasará a constituirse como el ideal del empleado comprometido con la supervivencia de la organización en este nuevo esquema. El campo de las identidades profesionales y ocupacionales en el que la cultura se relaciona con el saber adquirido en el trabajo o para el trabajo constituye un antecedente en el estudio del llamado managerialismo (Deetz, 1992). En sus orígenes, este enfoque fue estudiado en términos parsonianos de "modelos profesionales ideales" que servían de inspiración a los individuos en el proceso de elección profesional (Guadarrama, 2000: 221). Es así como desde Latinoamérica se comienza a converger con los postulados funcionalistas y managerialistas de la cultura corporativa norteamericana.

\section{Carácter ideológico de la cultura corporativa}

En el seno de la reconfiguración del poder en este nuevo marco global que pone el acento en la adaptación a las reglas de competitividad imperantes, De Gaulejac (2005) comienza a hablar de manera directa de una "ideología gestionaria", en el sentido que el management 
se va a presentar de una manera pragmática, desideologizada, fundada sobre el principio de eficacia y de utilidad más que sobre la pertinencia o la veracidad de las ideas que propone. De acuerdo al autor, su carácter ideológico radica en que constituye una construcción de un sistema de interpretación del mundo social que implica un orden de valores y una concepción de la acción. Apuntar a su carácter ideológico supone mostrar que detrás de las herramientas, los procedimientos, los dispositivos de información y de comunicación, todos éstos aparentemente neutros, se esconde una cierta visión del mundo y un sistema de creencias. En el mismo sentido, Chevallier (2005: 78) remitirá al carácter ideológico de la cultura corporativa como aquel que reposa sobre un sistema de creencias, sobre una cierta visión de las relaciones sociales al seno de los espacios de trabajo.

Por su parte, el carácter ideológico de la cultura de empresa es visto por Dujarier (2005) en la manera de prescribir la superación sin tregua del individuo. La exigencia hacia el empleado no tiene límites, "nunca estará a la altura de las circunstancias". Así, la calidad debe ser total, la rentabilidad debe crecer indefinidamente, la satisfacción del cliente debe ser absoluta. Este lenguaje prescriptivo es difícil que concite rechazo en los trabajadores debido a su nivel de abstracción, su contenido moralista y su radical alejamiento de un lenguaje que lleve a la acción. "Un sueño grande nos motiva a trabajar todos en la misma dirección para lograr ser la mejor empresa de bebidas (...) Trabajamos para reforzar el prestigio de nuestra compañía y de sus marcas con los más altos estándares de integridad", propone un folleto que busca tentar a jóvenes profesionales a las filas de la empresa de bebidas Quilmes (2011). Palabras con escasa densidad, que no representan nada desde el punto de vista de la actividad o la organización del trabajo. ¿Cómo oponerse a la calidad, la transparencia, la satisfacción, la cooperación, la integridad? Las abstracciones permiten la acumulación de mandatos sin que represente tensión alguna para quien debe acatarlos. Es necesario traducirlos en actos concretos para que aparezcan los límites y contradicciones que esconden.

La eufemización del conflicto lleva a que el término "despido" no se encuentre codificado dentro de las normas culturales de las empresas. Las frases elípticas que hacen mención a la falta de adaptación del asalariado a la cultura corporativa son claras muestras de esta realidad. Según el gerente de marketing de Great place to work (2011),

"cuando las grandes empresas no pueden evitar los despidos, las reducciones se manejan de manera tal que se preserva la confianza que los gerentes han forjado con sus empleados".

Para este gerente, es importante la manera en que la empresa transmite la información relacionada con "el proceso de reducción de personal”. ¿Cuál es la necesidad de invertir tantas energías para brindarles beneficios a los empleados despedidos? De acuerdo con el directivo de GPTW, estas medidas garantizan que los empleados que siguen en sus puestos de trabajo sepan que sus colegas están bien cuidados, lo que contribuye a mantener la confianza entre la empresa y los empleados. 
Para eliminar toda mención a las relaciones de poder y el conflicto inherente que éstas conllevan, Dujarier (2005: 105) señala que el discurso managerial apunta menos al trabajo y a su finalidad que al proceso mismo de trabajo: es más la dinámica que el resultado lo que cuenta. El proceso de trabajo managerial, traducido en términos de calidad, trazabilidad, responsabilización, control de costos, deviene más importante que el trabajo mismo. El buen desarrollo del proceso es lo que va a devenir la principal cuestión de la actividad laboral. Por esto, para poder observar el carácter conflictivo de la relación laboral, será necesario visibilizar lo que queda totalmente obturado en el trabajo gerencial: la actividad laboral y su finalidad debieran ser puestos en el centro del debate para observar la emergencia del conflicto. En suma, la despersonalización del poder -con la concomitante transformación de la empresa en una persona mítica- es lo que lleva a la abstracción de las prescripciones.

El trabajo de eufemización del conflicto está presente en toda la literatura managerialista, por lo que se hace imprescindible un trabajo de elucidación de lo que la noción de cultura corporativa esconde, dado que en ningún momento se parte de la puja de intereses que existe entre el capital y el trabajo. Es por eso que, a fin de cuentas, hablar de una cultura empresarial no es otra cosa que hablar de la ideología que intenta enmascarar los intereses contrapuestos para alinearlos en pos de los intereses de una sola de las partes.

Para lograr una mayor comprensión del carácter ideológico de la perspectiva de la cultura organizacional, se hace necesa- rio saber en qué consiste la "cultura corporativa" y cuál es su principal objetivo. En definitiva, el desafío esencial que busca la cultura de empresa siempre ha sido el de lograr que los empleados se identifiquen con la organización y consideren los éxitos de las empresa como propios. Se debe lograr la alineación voluntaria con los valores, la misión y la visión que la misma define. Para esto se necesita que la empresa se distinga por poseer una cultura "exitosa", por lo que ésta deberá hacer hincapié en el liderazgo encarnado en la "misión" de la empresa, tal como reza un folleto de la empresa agroalimentaria Cargill (2011) distribuido en una jornada de reclutamiento de jóvenes profesionales en la Universidad de San Andrés en Buenos Ares: "Tendrás la posibilidad de gestionar tu propia carrera, orientándola con cada una de tus elecciones".

La adhesión a la cultura de la empresa por propia iniciativa, se lograría a través de la delegación de responsabilidades a lo largo de toda la pirámide jerárquica con la consecuente asunción individual de los riesgos. El involucramiento de los empleados con la compañía estará condicionado por las necesidades del mercado que fungirá de guía para saber qué y cómo producir, destruyendo las barreras que impiden la innovación y el cambio (Rose, 1989: 118). Estas características redundarán en un beneficio económico para la empresa.

Para Great place to work (2011), la mejora en el "clima organizacional" impacta en el desempeño -la rentabilidadde las organizaciones por lo que es necesario implementar las prácticas que permiten mejorarlo. Los beneficios que reportaría un "excelente lugar para trabajar" 
(definido por la consultora como aquel donde "confío en la gente para quien trabajo, tengo orgullo por lo que hago y disfruto de compartir el trabajo con mis compañeros") serían "la atracción de los empleados más calificados, menores costos de rotación del personal, productos y servicios de mejor calidad y, por sobre todo, menores costos de servicio médico".

En suma, la retórica managerial presenta la adhesión a los valores y a la cultura de empresa así como la cooperación de los asalariados en términos de supervivencia del grupo y de cada uno de los empleados. La frontera entre un ellos -la dirección- y un nosotros -los asalariados- se ha desplazado hacia un ellos -las empresas competidoras a las que hay que vencer a cualquier precio -y un nosotros -la empresa- que sostiene la idea que cada uno debe luchar por salvar a la corporación (Philonenko, 1997).

\section{Doctrina de la excelencia: la vía "espiritual" de la conquista cultural}

El carácter ideológico de la perspectiva managerial de la cultura empresarial encuentra su origen en la difusión de las doctrinas de la excelencia (Rose, 1989; Smith, 1990) que resaltaban las figuras del emprendedor exitoso y del empleado de alto rendimiento que logra subordinar sus intereses individuales para el bien de la firma. Este tipo de trabajador podría ser cualquiera que se lo proponga si desarrolla sus capacidades de autorrealización, de presentación de sí y de la eficaz administración de su trayectoria profesional.

De aquí que durante la década del 80 del Siglo pasado, comiencen a estar en boga la contratación, por parte de las empresas, de consultores con formación psicológica, que operen en el terreno organizacional para entrenar a los empleados más calificados en el desarrollo de su sensibilidad, su confianza, su nivel de conciencia, entre otros. Éstos pueden ser formados en las técnicas para comprender sus motivaciones más íntimas y de quienes trabajan con ellos. Esta ruptura la encuentra el director de la consultora Great place to work:

Mientras en el pasado, el líder (...) tenía que resolver aspectos puramente técnicos, hoy, las organizaciones (...), demandan mucho más de él, o sea no alcanza con esto, entonces, tienen que sumarse mucho más a procesos más adaptativos; se les demanda (...) responsabilidad sobre el clima organizacional, se les demanda mucho más... (Great place to work, 2011).

De esta manera, se ha encontrado la intersección entre el éxito económico, el progreso en la carrera y el desarrollo personal, junto con el territorio perteneciente al mundo psicoterapéutico.

La publicación de dos best sellers norteamericanos inauguran la perspectiva culturalista de la empresa -El precio de la excelencia en 1981 y La pasión de la excelencia en 1985-, los cuales forjaron las premisas básicas de gobierno de la conducta de los individuos dentro de la organización y de la invención de sus identidades, y que forma parte de lo que Ibarra, (2000:265) llama la "literatura de la excelencia":

Las organizaciones deben construir un escenario que proporcione a los individuos un sentido figurado de ellos mismos como sujetos de excelencia, ha- 
ciéndolos responsables directos del destino de la organización; la introyección de esta imagen, que se funda en la exaltación de la perfección narcisista y la capacidad emprendedora, permitirá a las organizaciones aprovechar todas las energías del individuo, quien trabajará en los márgenes de sus capacidades con el único objeto de ser el mejor .

Peters y Waterman (1992) -autores de esos textos- presentan los casos de éxito de culturas de la excelencia de una manera peculiar:

Mientras trabajábamos en la investigación de nuestras compañías nos sorprendió el uso dominante de historias, cuentos y leyendas que utilizaban las personas para explicar las características de sus grandes instituciones. Todas las compañías que entrevistamos, desde Boeing hasta McDonald's, eran simplemente ricos tapices tejidos de anécdotas, mitos y cuentos de hadas. (...) La gran mayoría de las personas que cuentan historias sobre Watson de IBM nunca ha conocido al hombre, ni ha tenido experiencia directa de la realidad original y cotidiana que relatan. Sin embargo, en un sentido organizativo, estas historias, mitos y leyendas son importantes porque expresan los valores compartidos de la gestión empresarial o, lo que es lo mismo, de la cultura.

Las claves que presenta el discurso gerencial, de acuerdo a la literatura de la excelencia, se puede resumir en torno a seis ejes: la sobrevaloración de la acción, el mito del éxito, la competencia por la pertenencia a una élite, la adaptabilidad permanente, la equivalencia entre progreso económico y progreso social y la conciliación del interés individual con el de la empresa. Se trata de adherir a estos principios o de ser excluido, no hay una tercera vía.

Ante la poca claridad y especificidad de la idea de excelencia, la traducción operativa queda resguardada de críticas. La aplicación de estándares de desempeño (muchas veces con criterios subjetivos), los procedimientos de evaluación y los sistemas de recompensa, se ven grandemente facilitados. La exaltación del individuo como el ideal de la organización (y su correspondiente exigencia de iniciativa, proactividad, capacidad de trabajo bajo presión, compromiso para con los objetivos organizacionales) fue funcional con las grandes reestructuraciones que se produjeron durante las décadas del 80 y 90 .

Es interesante la formación de subjetividades dentro del mundo de las empresas, ya que las diferentes técnicas de motivación, la gestión participativa y la cultura del liderazgo no son sino tecnologías de gobierno de los sujetos que permiten que las cosas se hagan. Las nuevas prácticas laborales tienden a conformar un nuevo tipo de subjetivación, en la cual la nueva identidad que se pretende construir es la del hombre libre que se hace cargo de sí mismo. En este marco, la figura del manager es paradigmática, ya que éste constituye el ideal del trabajador autónomo de la empresa, al cual todos deben aspirar. El compromiso introyectado por los empleados, aceptando los indicadores de la excelencia, genera que cualquier necesidad de ajuste se funde en que son los empleados los que deben correr con la responsabilidad de los fracasos. La razón de estos traspiés radicaría en que los empleados no estuvieron 
suficientemente alineados con la cultura corporativa $^{3}$.

La literatura managerial se alinea con el discurso que destila la cultura corporativa, que busca obtener no tanto la sumisión dócil como la adhesión voluntaria. Todas las estrategias se centrarán en promover una cultura de la diversidad y el respeto por el otro, en la cual cada uno se hace cargo de la construcción de su trayectoria de manera autónoma.

Para lograr este cometido, la empresa necesita basarse en un discurso que concite credibilidad en sus empleados. De esta manera, la firma se encargará de dotar de sentido al trabajo en la organización a través de apelaciones al bien común. Todo esto necesitará de la activación de una aparato de producción ideológica como elemento esencial de su funcionamiento y sustentado en dos motivaciones principales: 1) mostrar una imagen de sí misma como elemento determinante de su política comercial (el marketing abogará por mantener la coherencia entre esta imagen y los productos que coloca en el mercado) y, 2) ofreciendo un apoyo productor de sentido que canalice las esperanzas y los deseos, calmando las angustias y las dudas y ofreciendo a sus agentes una razón de vivir (Aubert y De Gaulejac, 1979: 112 y 113).

La eficacia de este aparato llamado "la ideología corporativa" reside en que logra que el trabajador no se sienta instrumentalizado por una relación laboral opresiva, sino que tenga el sentimiento de ser un sujeto que trabaja por su propia cuenta -lo que Smith (1990: 24) llama "autonomía coercitiva”-. Esta conformidad conduce al individuo a encarnar el sistema de valores de la empresa y a ser reconocido de acuerdo a los símbolos del éxito y la excelencia. La producción de la excelencia combina éxito en términos materiales con inversión en términos espirituales. La búsqueda de una perfección sin límites forma parte de esta ecuación que necesita ser legitimada con apelaciones de raíz simbólica.

Sin embargo, la doctrina de la excelencia encuentra serias contradicciones que no logran ser saldadas. La "preocupación" de las empresas por la cultura no puede ser entendida de manera separada respecto de las contradicciones del propio capitalismo (Bell, 2006). El interés individual por la autorrealización debió conjugarse con el aumento de la competencia y las demandas crecientes de los consumidores. Como Rose (1989) sostiene, el imperativo psicológico del trabajo pasó a ser tan importante como el económico. Esto implicó que la cultura deba integrar lo simbólico con lo racional y estratégico, proponiendo administrar lo simbólico para controlar lo inmanejable -es decir, las fluctuaciones del mercado y de la demanda-. La preocupación por la búsqueda de una cultura fuerte y unificada es que ésta genera un mayor retorno a las inversiones (Saffold citado por Braga, 1996), ya que permite "alinear" a los empleados en épocas de crisis. La socializa-

3 Sería interesante indagar acerca de los fundamentos de la obediencia y aceptación voluntaria de la subordinación incluso a costa de la propia seguridad personal. 
ción centrada en torno a los valores organizacionales ofrecería una solución para controlar la incertidumbre "interna" derivada de las idiosincrasias (Pascale citado por Braga, 1996) y "externa" derivada de los mercados.

Tal como sostienen Bourdieu (1998) y Foucault (1978), la cultura que unifica es también la misma que separa, ya que ésta es intrínsecamente divisiva y descansa sobre relaciones de poder. Cuando el discurso corporativo promueve el individualismo y simultáneamente el espíritu de equipo y de comunidad integrada, genera contradicciones que necesitan de una resolución, al menos precaria y transitoria. Lipovetzky citado por López (2004), va a apelar al concepto de ensemble individualism o individualismo conjunto para evocar "la trampa de la razón pos-moralista" en la cual las pasiones individualistas de autonomía y realización personal son estimuladas para cumplir eficazmente con los objetivos de competitividad de las empresas. Así, por ejemplo, el trabajo en equipo será valorizado porque es el grupo quien permite al individuo desarrollarse, mejorando en el mismo proceso la eficacia de la empresa. Pero esto de ninguna manera resuelve las contradicciones culturales de la retórica empresarial.

\section{Confianza y cultura}

Las contradicciones que genera la cultura de empresa y los intentos por desanudarlas llevan a que la alta dirección deposite en sus empleados una alta dosis de confianza, pero la incertidumbre que encierra confiar en una relación que se presenta como necesariamente conflicti- va, hace que las compañías se sirvan de mecanismos de medición del clima organizacional que logren brindar un panorama más previsible de la relación entre el salariado y el empleador.

La consultora Great Place to Work lleva a cabo una evaluación y comparación de las prácticas culturales en diferentes empresas a través de 115,000 encuestas llevadas a cabo a todas las escalas de la jerarquía organizacional. Esto se plasma en un estudio sobre los beneficios generados a través de la confianza en los llamados "excelentes lugares de trabajo", con la correspondiente elaboración de un Trust Index (índice de confianza). De acuerdo con esta consultora, existen cinco dimensiones de un "Excelente lugar para trabajar" (Cuadro 1).

Cada una de las dimensiones constituye un atributo que los asalariados -y no la empresa, de acuerdo con esta consultora- define como valioso. Los primeros tres, credibilidad, respeto e imparcialidad, se corresponden con el grado de confianza alcanzado por los jefes en relación con sus empleados.

Tal como lo mencionamos, el Trust Index se realiza por medio de una encuesta de clima organizacional, que proyecta resultados en términos cuantitativos -promedio de confianza-. Esto es cotejado con un cuestionario realizado a los directivos llamado culture audit, en el que se examinan las prácticas y políticas de la organización. A la encuesta de confianza se le otorga un peso de dos tercios de la nota final para la compañía, mientras que para la auditoría cultural, se le asigna un tercio de la respectiva nota. Como resultado, se elabora un ranking de todas las empresas estudiadas que se difunde en 


\begin{tabular}{|c|c|c|}
\hline \multicolumn{3}{|c|}{$\begin{array}{c}\text { Cuadro } 1 \\
\text { Dimensiones y subdimensiones de un "Excelente lugar para trabajar" }\end{array}$} \\
\hline Dimensión & Subdimensiones & \\
\hline Credibilidad & $\begin{array}{l}\text { - Comunicaciones abiertas y accesibles } \\
\text { - Capacidad para coordinar recursos humanos y materiales } \\
\text { - Integridad al impulsar consistentemente la misión }\end{array}$ & CONFIANZA \\
\hline Respeto & $\begin{array}{l}\text { - Reconocimiento y apoyo al desarrollo profesional } \\
\text { - Participación en decisiones relevantes } \\
\text { - Cuidado de los empleados como individuos con vida } \\
\text { e intereses personales }\end{array}$ & \\
\hline Imparcialidad & $\begin{array}{l}\text { - Equidad - tratamiento equitativo para todos en términos } \\
\text { de recompensa y esfuerzos } \\
\text { - Ausencia de favoritismo al contratar y promover } \\
\text { - Justicia - ausencia de discriminación, apelación }\end{array}$ & \\
\hline Orgullo & $\begin{array}{l}\text { - Por el trabajo personal desempeñado } \\
\text { - Por el propio equipo de trabajo y sus logros } \\
\text { - Por la empresa, sus productos y su presencia en la comu- } \\
\text { nidad }\end{array}$ & \\
\hline Camaradería & $\begin{array}{l}\text { - Posibilidad de ser auténtico } \\
\text { - Socialmente amigable con atmósfera de bienvenida } \\
\text { - Sentido de familia o equipo }\end{array}$ & \\
\hline
\end{tabular}

Fuente: Great Place to Work (2011).

los medios gráficos más importantes del país, dentro de los suplementos especializados.

El servicio que presta esta consultora es "la generación de confianza en los lugares de trabajo". Así lo constata la directora del departamento de investigación corporativa de Great place to work (2011),

en lugares de trabajo con índices altos de confianza, los empleados muestran mayores niveles de cooperación (...), así como mayores niveles de compromiso con su trabajo, con el trabajo de la organización y con la visión de los directivos de la organización.
Esto daría cuenta de la "calidad" de su cultura. En efecto, un alto grado de confianza redundaría en un alto espíritu de cooperación y un profundo sentido de compromiso con la misión, visión y valores de la firma. La consultora promete resultados concretos en estos lugares de trabajo "ideales": un desempeño financiero a largo plazo más sólido, menor rotación de personal en relación con sus pares dentro de la industria (la cuestión de la retención del "talento" es una preocupación mayúscula en las grandes corporaciones), más solicitudes de empleo que sus pares, una fuerza laboral integra- 
da en la que diversos grupos de personas crean y contribuyan a una cultura laboral común de la cual todos se beneficiarían. De acuerdo a sus predicciones, el impacto financiero de la confianza, la cooperación y el compromiso, sería insoslayable:

una gran confianza trae consigo fluidez en la colaboración y el intercambio de ideas, confianza en la visión de futuro de los directivos y fe en la imparcialidad fundamental con que serán tratadas las personas, características estas que contribuyen (...) a la producción que hacen exitosa a una empresa (Great place to work, 2011).

Great place to work (2011) presenta a Microsoft como un "campeón cultural". El director de recursos humanos de la firma sostiene que la confianza es la base sobre la que está construida la cultura de su organización:

La cultura de Microsoft se construye en torno a nuestra misión de ayudar a la gente a alcanzar su máximo potencial (...). Uno puede diferenciar a los empleados de Microsoft en todo el mundo por su mentalidad ganadora e innovadora, su deseo de superación permanente y de tomar grandes desafíos, y su aspiración a transformar la realidad a través de la tecnología (Great place to work, 2011).

En suma, la confianza constituye la base sobre la que se sustentará el intento de buscar una cultura homogénea que englobe a todos los asalariados por igual. Pero los esfuerzos que realiza la empresa no sólo para ganarse la credibilidad de sus trabajadores sino también para auditar el grado de éxito de estas inversiones, no son otra cosa que el anverso y el reverso de una misma realidad que se muestra siempre conflictiva, aún en grado de latencia.

\section{Grado de alineamiento del asalariado con la cultura de la empresa: la gestión del "talento"}

En un fascículo dedicado a cuestiones de recursos humanos dentro de la colección "Master en negocios" que publica el suplemento económico del diario argentino Clarín, Simón (2009), profesora en posgrados de gestión de recursos humanos, sostiene que una de las funciones principales de un departamento de recursos humanos es la de proponer una serie de principios rectores, una suerte de guía o pauta de comportamiento, que se nutren de la "cultura de la empresa". Estos principios se sustentan en un alto grado de abstracción, puesto que "recogen las intenciones de la alta dirección en cuanto a la gestión de su talento y a la manera de capitalizarlo para contribuir a los resultados del negocio". Esto se logrará a través de la internalización de las presunciones básicas subyacentes (Schein, 1995) enraizadas en la cultura de la firma.

En este sentido, si la empresa pretende transitar hacia lo que la autora llama "cultura del rendimiento", ésta debe estar articulada con prácticas específicas de recursos humanos que generen alguna forma de medición del grado de adhesión del empleado con dicha cultura -como evaluaciones de desempeño, retribución por resultados- que den sustento concreto y operacionalizable a dicha cultura. Evocando el ahorro en los costos de control del personal que supone una cultura corporativa consolidada e internali- 
zada en cada uno de sus miembros, Simón (2009: 22) describe de manera axiomática lo que en esencia es la cultura: "lo que los empleados hacen cuando el jefe no está en la oficina" (Simón, 2009: 22).

El despliegue de supuestos compartidos y patrones de comportamiento garantiza que los trabajadores desarrollarán su labor dentro de un marco de referencia concreto sin necesidad de excesivos controles. La manera de asegurarse que el asalariado se encuentre alineado con la visión, las metas y los objetivos de la organización-componentes esenciales de la cultura desde las perspectivas managerialistas- así como con los medios que hay que utilizar para lograrlos, se encuentran en las evaluaciones de desempeño, las cuales fijarán criterios que sirvan de reaseguro de dicho alineamiento.

Una de las novedosas maneras que encontró la firma argentina Tenaris de articular la cultura con resultados concretos del negocio es la implementación de los "Premios Valores" (donde se premia a los que han implementado de la manera más eficaz los postulados de la mejora continua enarbolados diariamente por la empresa) - en su boletín "Flash" (2011) distribuido a todos los empleados de la Argentina- evento en el cual se recompensa a quienes "promuevan la calidad, la seguridad y el trabajo en equipo desde la perspectiva de la mejora continua que hace a la cultura de la empresa y estén en línea con el proyecto 00100 (cero accidentes, cero defectos ciento por ciento cumplimiento)".

La gestión del encuadramiento y la alineación con la cultura y los objetivos de la firma, necesita de un cuidado especial a la hora de contratar empleados. No se puede reclutar personas carentes de los "talentos" requeridos por la organización. Es por eso que las compañías pondrán todas sus energías en captar a los candidatos más aptos gestionando sus aptitudes a través de evaluaciones periódicas. La obsesión que embriaga a los responsables de diseñar las políticas de recursos humanos de las grandes firmas es la búsqueda de líderes potenciales. Es por eso que la gestión del "talento" se asemeja mucho a una administración del encuadramiento y la movilización de los trabajadores. El tema del reclutamiento del personal que se encuadre dentro de la cultura específica de la empresa preocupa cada vez más a las empresas (aún cuando es dudoso que lo logren), al menos es lo que el director actual de la consultora Great Place to Work (2011) expresa en su página web institucional respecto de la última variable que incluyeron en la encuesta:

(...) La incorporación (...) de integrantes al equipo que se adecuan a la cultura de la organización (...) No les preguntamos si son competentes o no, les decimos cómo (...) [el] empleado ve a su jefe que, en términos de comportamiento, (...) está incorporando gente al equipo que se adecuan a la cultura de esa organización. Se debe a que se detecta que en el comportamiento que hace un "excelente lugar para trabajar", la decisión de un gerente, de un líder, en cuanto a incorporar personas e integrarlas a un equipo y saber sopesar los aspectos culturales, más que las competencias técnicas, es estratégico y es clave.

¿Qué principios se suelen utilizar para medir la potencialidad de la adhesión a la cultura de la empresa por parte 
del candidato -talento- a ser contratado por la empresa? Precisando los principios de contratación de Google (citado por Great place to work, 2011), una de las empresas que el mundo corporativo en general y la consultora mencionada en particular, consideran como el benchmark -el modelo a imitar- en términos de filosofía de gestión de sus empleados, que se instaló en la Argentina hace unos poco años. Esta filosofía bien puede representar la actual política de contratación de los departamentos de recursos humanos de las grandes empresas transnacionales:

Contratar: candidatos individuales consistentes con nuestra guía de lo que debe y no debe hacerse; candidatos individuales que tengan intereses y realicen actividades que "salten a la vista" $y$ que te produzcan ganas de hablar con ellos; líderes y líderes potenciales con voluntad comprobada para "ponerse manos a la obra" y cumplir objetivos; evitar contratar especialistas con capacidades limitadas; en lugar de ello, contratar: generalistas con experiencia pertinente y capacidad de escuchar; personas para cargos que ellas puedan desempeñar mejor; no contratar personas de urgencia. Una buena contratación requiere tiempo.

El pragmatismo, el llamado a la acción y la pasión por el trabajo, son los ejes alrededor de las cuales giran estos preceptos.

Pero más interesante es la lista que esta empresa ha preparado para demarcar de lo que debe y no debe hacerse:

Contratar personas: que sean más inteligentes y entendidas que usted; que crecerán con su equipo y con la empre- sa; que le agreguen valor al producto y a nuestra cultura; polifacéticas con intereses y talentos únicos; que cumplirán con los objetivos; con sentido ético y que se comuniquen abiertamente; entusiastas, automotivadas y apasionadas; sólo cuando haya encontrado a un excelente candidato; que inspiren a otras y que trabajen bien con los demás; No contratar personas: manipuladoras $\mathrm{O}$ con mucha conciencia política; que sólo quieran un empleo; que vivan sólo para trabajar; que sólo piensen en problemas; con destrezas o intereses limitados; que no contribuirán bien a ambos; que prefieran trabajar solas; de las cuales no pueda aprender o que no le supongan un desafío.

De acuerdo con estos postulados -y con los del capitalismo en general-, el éxito material va de la mano con la inversión espiritual. La búsqueda de la perfección moral debe conducir necesariamente al éxito. El talento, la posibilidad de agregar valor, la realización personal es lo que debe buscarse. Pero esta vida no es para cualquiera. Aquí no hay lugar para los "débiles", o al menos para aquellos que tengan conciencia de su debilidad. Los conflictos internos que viva el individuo no deben ser visibilizados. Incluso este "superhombre" no debe tener conciencia de su misma condición de asalariado. Pareciera ser que el trabajador ideal es aquel que guarda una devoción y una entrega total con la compañía, logrando soslayar cualquier conflicto, transformándolo en energía positiva. El carácter ideológico de estos mandatos reside en que constituyen un sistema de creencias -en fin, una "cultura"- que jerarquiza a los sujetos de acuerdo con carac- 
terísticas psicológicas, lo que redundará en una gestión híbrida y dual de la mano de obra, dividida entre los que han logrado incorporar la cultura de la empresa y los que tarde o temprano se expondrán al estancamiento de sus carrera o directamente a la desvinculación de la compañía.

\section{Conclusiones}

La finalidad última de las corrientes de pensamiento que convergen en lo que se suele llamar "la cultura corporativa" es lograr el consentimiento de sus empleados. Pero ¿hasta qué punto el sujeto incorpora significados y actúa con el fin de reforzarlos? El sujeto también puede decidir no comprometerse con la cultura ya sea a través de la salida de la organización o por medio de formas sutiles de resistencia ¿En qué medida puede o logra una adhesión pragmática, oportunista o distanciada de los significados propuestos por la cultura? La estrategia según la cual una cultura se define como dominante forma parte de un proceso político de imposición de significados supuestamente compartidos. En dicho proceso, las culturas dominantes definen quiénes formarán parte de la cultura dominante -corporativa- y quiénes estarán condenados al ostracismo y la posibilidad de una eventual desvinculación de la empresa.

En su intento de crear una unidad en la definición de la realidad, la cultura dominante crea una jerarquía del mundo social; no sólo define qué son y qué deben ser las personas, sino que también hace y deshace grupos, creando insiders y outsiders. Usa su poder simbólico para santificar, pero también para sancionar, mostrando a la persona cuál es su lugar y cuánto valen. El poder simbólico de lo que se suele llamar "cultura corporativa" radica en que es capaz de imponer una estructura de clasificaciones como natural, de una manera consensuada. Así, es posible compartir la visión comúnmente aceptada de la Sociología del trabajo -crítica- que una característica intrínseca del poder simbólico es que su efectividad mana del hecho que usualmente se presenta a la audiencia de una manera conciliadora.

Esto permite al poder imponer clasificaciones y categorizaciones bajo la apariencia de taxonomías religiosas y filosóficas. La efectividad de estas clasificaciones depende de actos de poder específicos tales como los mecanismos de recompensa y castigo. Una manera de que estas clasificaciones aparezcan como naturales es creando ceremonias y rituales de confirmación, tales como las evaluaciones $360^{\circ}$ que se realizan a los managers periódicamente en las que intervienen jefes, subordinados, pares y hasta clientes externos a la organización. Los rituales como éste tienen el efecto de organizar el orden establecido, confirmando quién debe ejercer el poder y quién debe obedecer. Sus funciones residen en reforzar los valores organizacionales estableciendo distinciones y determinando quién está adentro y afuera, pero también se basan en crear grados de inclusión de acuerdo con la proximidad al grupo de los elegidos.

Sin embargo, el poder no define a la identidad del sujeto de una manera inexorable. Las ideas no manejan a otras ideas de la misma manera en que los hombres manejan a otros hombres. Del 
sistema de relaciones de fuerza no puede deducirse automáticamente la clave de lectura de las relaciones de dominación simbólica. La capacidad de agencia descansa en que el individuo es capaz de negociar su involucramiento con la empresa; puede estar comprometido con el trabajo pero no con la organización o con ciertas dimensiones del espacio de trabajo (por ejemplo, con colegas), pero no con el trabajo mismo en la firma.

Los espacios de socialización por excelencia en el intento de manipular símbolos y mitos los constituyen los programas de capacitación ejecutiva, arena en la cual no sólo se trata de controlar sino también de generar consentimiento. A través de un aparato de técnicas de recursos humanos, se sustrae al empleado de su trabajo cotidiano con la finalidad de producir y reproducir compromiso en ellos. La participación en ámbitos colectivos es utilizada por los altos directivos no sólo para controlar a sus subordinados, sino también para contener los conflictos que puedan emerger y así minimizar las resistencias.

En suma, el carácter eminentemente político de la cultura de empresa (Wright, 1998) implica que la retórica que la suele caracterizar está directamente comprometida con el ejercicio del poder, que a su vez produce dos efectos contrapuestos: por un lado, la introyección del dispositivo managerial, entendiendo a éste como la forma que encuentra el poder de legitimarse a sí mismo a través de apelaciones al bien común de los trabajadores y la aceptación consentida de las coacciones en el trabajo por parte de los asalariados. Por otro lado, la capacidad de agencia del individuo que genera cierta tensión en este proceso.
En rigor, las teorías managerialistas basadas en el control por medio de la manipulación de la cultura deberán enfrentarse con relaciones asimétricas de poder al interior de las organizaciones, lo que conducirá inexorablemente a desnudar el carácter violento (si bien sutil) de todos los intentos de imponer una cultura única en un espacio de trabajo signado por una multiplicidad de interpretaciones de la realidad de la empresa. Esto nos convoca a retomar los postulados clásicos de la Sociología del trabajo para analizar críticamente la irrupción de corrientes funcionalistas y etnocéntricas de la cultura corporativa -provenientes de los centros del poder económico mundial- en los espacios de trabajo de nuestra región.

\section{Referencias Bibliográficas}

Abramo, Lais y Montero, Cecilia (2000). Origen y evolución de la sociología del trabajo en América Latina. En: De la Garza, Enrique (ed.) (2000). Tratado latinoamericano de sociología del trabajo. México DF, México, Fondo de Cultura Económica.

Aubert, Nicole y De Gaulejac, Vincent (1979). Le cout de l'excellence. París, Francia, Éditions du seuil.

Bell, Daniel (2006). Las contradicciones culturales del capitalismo. Madrid, España, Alianza.

Bourdieu, Pierre (1998). La distinción. Criterios sociales del gusto. Madrid, España, Taurus.

Braga Rodrigues, Suzana (1996). Corporate cultures and de-institutionalization: implications for identity in a Brazilian telecommunications company. En Palmer, Gill y Clegg, Stewart (1996). Constituting management: mar- 
kets, meanings and identities. Berlín, Alemania, De Gruyter.

Brown, James (1982). La psicología social en la industria. México DF, México, Fondo de Cultura Económica.

Calas, Marta y Smircich, Linda (1992). "Using the ' $F$ ' word: feminist theories and the social consequences of organizational research". En Mills, Albert y Tancred, Peta (eds.) (1992). Gendering Organizational Analysis. Londres, Inglaterra, Sage.

Cargill, S.A. (2011). Folleto institucional. Día de empresas. Buenos Aires, Argentina, Universidad de San Andrés.

Chevallier, Marjorie (2005). La politique de l'épargne salariale au service du consentement au travail. En Le Floch, Marie Christine (ed.) (2005). La Question du consentement au travail: De la servitude volontaire à l'implication contrainte. París, Francia, L'Harmattan.

De Gaulejac, Vincent (2005). La société malade de la gestion: idéologie gestionnaire, pouvoir managérial et harcèlement social. París, Francia, Éditions du seuil.

Deal, Terence y Kennedy, Allan (1982). Culturas corporativas: Ritos y rituales de la vida organizacional. México DF, México, Legis.

Deetz, Stanley (1992). Democracy in an age of corporate colonization, Developments in communication and the politics of everyday life. Nueva York, Estados Unidos, State University of New York Press.

Dujarier, Michel (2005). La soumission à la prescription de toute-puissance. En Le Floch, Marie Christine (ed.) (2005).

La Question du consentement au travail: De la servitude volontaire à I'implication contrainte. París, Francia, L'Harmattan.
Fígari, Claudia (2007). Competencias corporativas, mejora continua y pedagogía empresaria: crítica al patrón normalizador/evaluador en el orden laboral y profesional. V Congreso Latinoamericano de Sociología del Trabajo. Montevideo, Uruguay, 18 al 20 de abril.

Flash, (10-2011). Premios valores. Tenaris, Buenos Aires, Argentina.

Foucault, Michel (1978). Vigilar y castigar: el nacimiento de la prisión. México DF, México, Editorial Siglo XXI.

Gasparini, William (2005). Dispositif managérial et dispositions sociales au consentement. L'exemple du travail de vente d'articles de sport. En Le Floch, Marie Christine (ed.) (2005). La Question du consentement au travail: De la servitude volontaire à I'implication contrainte. París, Francia, L'Harmattan.

Great Place to work, [2011]. [Documento electrónico] [http:Ilwww.gptw.com] [sin compaginación] [Consultado el 2011-2011].

Guadarrama Olivera, Rocío (2000). La cultura laboral. En De la Garza, Enrique (ed.) (2000). Tratado latinoamericano de sociología del trabajo. México DF, México, Fondo de Cultura Económica.

Hughes, Evereth (1958). Men and their work. Nueva York, Estados Unidos, Free Press.

Ibarra Colado, Eduardo (2000). Teoría de la organización, mapa conceptual de un territorio en disputa. En De la Garza, Enrique (ed.) (2000). Tratado latinoamericano de sociología del trabajo. México DF, México, Fondo de Cultura Económica.

La Boétie, Étienne De (1993). Discours de la servitude volontaire. París, Francia, Payot.

Lipovetsky, Gilles (1994). El crepúsculo del deber: la ética indolora de los nue- 
vos tiempos democráticos. Barcelona, España, Anagrama.

López Pintor, Rafael (1986). Sociología industrial. Madrid, España, Alianza.

López Ruiz, Osvaldo (2004). O "ethos" dos executivos das transnacionais e o espírito do capitalismo. Tesis de doctorado defendida en la Universidad estadual de Campinas. San Pablo, Brasil.

Pascale, Richard (1985). The paradox of corporate culture: reconciling ourselves to socialization. California management review. Año 27, Vol. 27, $n^{\circ} 2$, pp. 24-41.

Peters, Tom y Waterman, Robert (1992). En busca de la Excelencia. Bogotá, Colombia, Editorial Norma.

Philonenko, Grégorie (1997). Au carrefour de I'exploitation. París, Francia, Édition Desclée de Brower.

Quilmes, Sociedad Anónima (2011). Folleto institucional. Día de empresas. Buenos Aires, Argentina, Universidad de San Andrés.

Rose, Nicholas (1989). Governing the soul: The shaping of the private self. Londres, Inglaterra, Free Association Books.
Saffold III, Guy (1988). Culture traits, strength, and organizational performance: moving beyond strong culture. Academy of management review. Año 12, Vol. $13, n^{\circ} 4$, Nueva York, Estados Unidos, pp. 546-558.

Schein, Edgar (1995). Cultura empresarial y liderazgo. Barcelona, España, Plaza \& Janes Editores.

Simón, Cristina (2009). La función de recursos humanos: misión, evolución y perspectivas. En Sbdar, Manuel (ed.) (2009). Capital humano: las personas con lo más importante. Pero, ¿quiénes, cómo, cuándo y cuánto? Buenos Aires, Argentina, Arte gráfico editorial argentino.

Smith, Vicki (1990). Managing in the corporate interest. Oxford, Estados Unidos, California University Press.

Sztulwark, Sebastián (2002). El estructuralismo latinoamericano: crisis, renovación y nuevos desafíos. Buenos Aires, Argentina, Prometeo.

Watson, Tony (1987). Sociology, work and industry. Londres, Inglaterra, Routledge.

Wright, Susan (1998). "La politización de la cultura". Anthropology today. Vol. 14, $\mathrm{n}^{\circ} 1$, febrero de 1998, pp. 7-15. 